\title{
Localized Induction Equation for Stretched Vortex Filament
}

\author{
Kimiaki KONNO ${ }^{\dagger}$ and Hiroshi KAKUHATA $\ddagger$ \\ $\dagger$ Department of Physics, College of Science and Technology, Nihon University, \\ Tokyo 101-8308, Japan \\ E-mail:konno@phys.cst.nihon-u.ac.jp \\ ‡ Toyama University, Toyama 930-8555, Japan \\ E-mail:kakuhata@iis.toyama-u.ac.jp
}

Received October 05, 2005, in final form February 16, 2006; Published online March 02, 2006

Original article is available at http://www.emis.de/journals/SIGMA/2006/Paper032/

\begin{abstract}
We study numerically the motion of the stretched vortex filaments by using the localized induction equation with the stretch and that without the stretch.
\end{abstract}

Key words: localized induction equation; stretch; vortex filament

2000 Mathematics Subject Classification: 35Q51; 37K15; 76B29

\section{Introduction}

Konno and Kakuhata are interested in motion of the stretched vortex filaments where the filaments are described by the localized induction equation (LIE) [1]. LIE is given by

$$
\boldsymbol{X}_{t}=\boldsymbol{X}_{s} \times \boldsymbol{X}_{s s},
$$

in which $\boldsymbol{X}$ is the position vector $(X, Y, Z), s$ is the arclength and $t$ is the time. This LIE was first obtained by Arms and Hama for the thin vortex filament with the localized induction approximation [2]. Their original equation is derived as

$$
\boldsymbol{X}_{t}=\frac{\boldsymbol{X}_{s} \times \boldsymbol{X}_{s s}}{\left|\boldsymbol{X}_{s}\right|^{3}}
$$

Under the condition $\left|\boldsymbol{X}_{s}\right|=1$, (2) is reduced to (1). $\left|\boldsymbol{X}_{s}\right|$ is equal to the local stretch of the filament $l_{s}$ defined by [3]

$$
l_{s}=\frac{\mathrm{d} l}{\mathrm{~d} s}=\left|\boldsymbol{X}_{s}\right|,
$$

where $l$ is the length along the filament. If $l_{s}=1$, there is no stretch, if $l_{s}>1$, stretch and if $l_{s}<1$, then shrink.

If there is no stretch, one can define the tangent vector $\boldsymbol{t}=\partial \boldsymbol{X} / \partial s$ with its magnitude 1 and use the Frenet-Serret scheme. Then one introduces the curvature and the torsion, and shows that equation (1) is equivalent to the nonlinear Schrödinger equation through the Hashimoto transformation [4]. For $l_{s} \neq 1 s$ does not mean the arclength but the parameter along the filament. LIE is an integrable equation. Moreover, we have shown that (2) is integrable and has a hierarchy which is constructed by the recursion operators [5].

In this paper, in order to compare (2) with (1) we will try to solve an initial value problem with the same initial vortex filaments. The numerical scheme is given in [6].

In the next section we show the numerical method for (2). In Section 3 numerical results are given. The last section presents conclusion. 

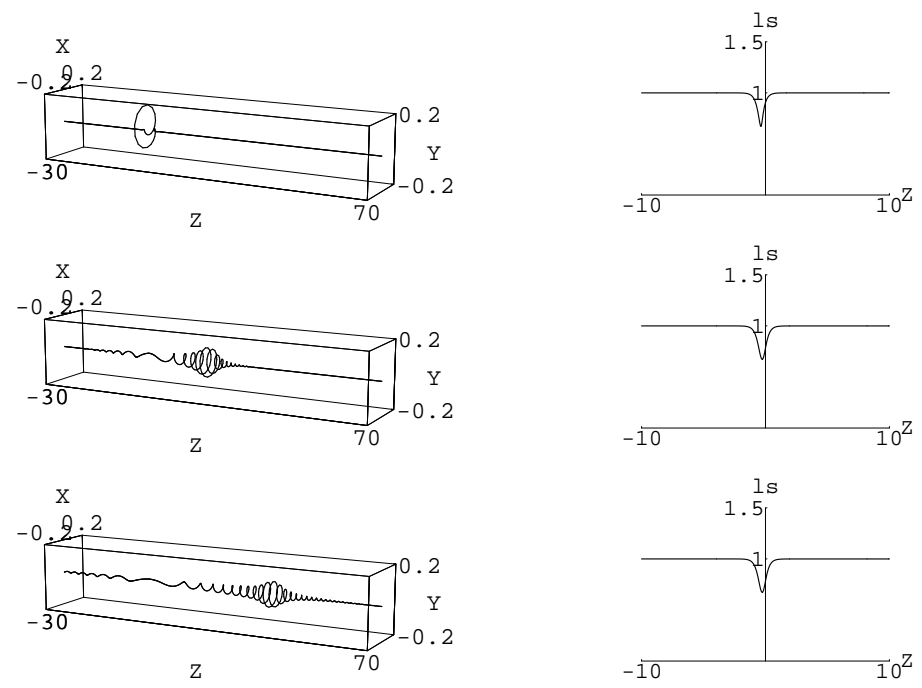

Figure 1. Time evolution (left) and local stretch (right) for the stretched vortex soliton (9) of (1) at $t=0,3,6$ for $A=0.6$ and $\lambda=1.5+\mathrm{i}$.

\section{Numerical method}

In order to solve (2), we use the following implicit finite difference equation [6], as an example, for the $x$ component as

$$
\begin{aligned}
\frac{X_{j+1, i}-X_{j, i}}{\Delta t}= & \frac{1}{L_{s}^{3}}\left[\frac{Y_{j, i+1}-Y_{j, i}}{\Delta s} \frac{Z_{j+1, I+1}-2 Z_{j+1, i}+Z_{j+1, i-1}}{\Delta s^{2}}\right. \\
& \left.-\frac{Z_{j, i+1}-Z_{j, i}}{\Delta s} \frac{Y_{j+1, i+1}-2 Y_{j+1, i}+Y_{j+1, i-1}}{\Delta s^{2}}\right],
\end{aligned}
$$

where

$$
L_{s}=\sqrt{\frac{\left(X_{j, i+1}-X_{j, i}\right)^{2}+\left(Y_{j, i+1}-Y_{j, i}\right)^{2}+\left(Z_{j, i+1}-Z_{j, i}\right)^{2}}{\Delta s^{2}}} .
$$

The subscripts $j$ and $i$ refer to the time $t=j \Delta t$ and $s=i \Delta s$, respectively. (3) and $Y$ and $Z$ components can be written as the following matrix form

$$
D_{j, i} H_{j+1, i+1}+\left(1-2 D_{j, i}\right) H_{j+1, i}+D_{j, i} H_{j+1, i-1}=H_{j, i},
$$

where

$$
H_{j, i}=\left(\begin{array}{c}
X_{j, i} \\
Y_{j, i} \\
Z_{j, i}
\end{array}\right), \quad D_{j, i}=\left(\begin{array}{lll}
0 & c_{j, i} & -b_{j, i} \\
-c_{j, i} & 0 & a_{j, i} \\
b_{j, i} & -a_{j, i} & 0
\end{array}\right)
$$

and

$$
\begin{aligned}
& a_{j, i}=\frac{\Delta t}{L_{s}^{3} \Delta s^{3}}\left(X_{j, i+1}-X_{j, i}\right), \quad b_{j, i}=\frac{\Delta t}{L_{s}^{3} \Delta s^{3}}\left(Y_{j, i+1}-Y_{j, i}\right), \\
& c_{j, i}=\frac{\Delta t}{L_{s}^{3} \Delta s^{3}}\left(Z_{j, i+1}-Z_{j, i}\right) .
\end{aligned}
$$

To solve (4), we introduce a recurrence formula

$$
H_{j+1, i}=E_{j, i} H_{j+1, i+1}+F_{j, i}
$$



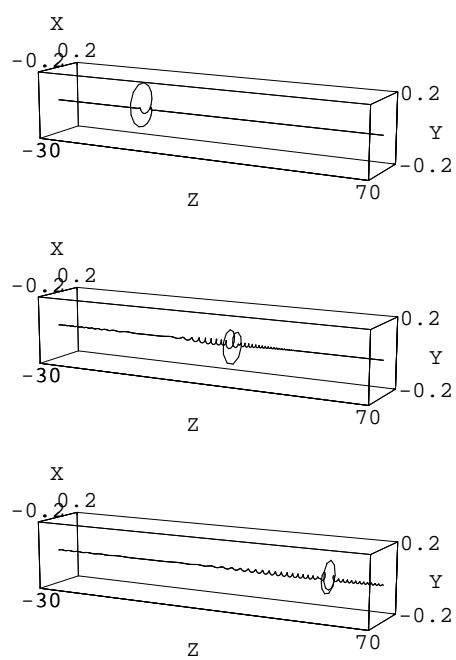
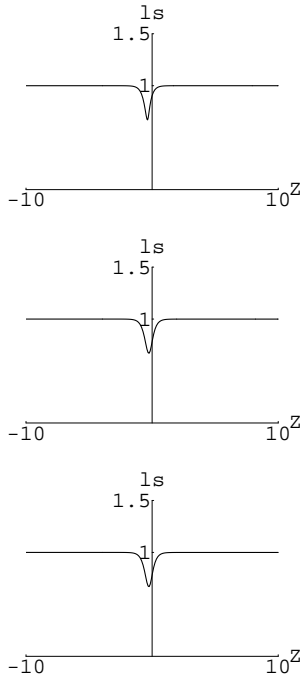

Figure 2. Time evolution (left) and the local stretch (right) for the stretched vortex soliton (9) of (2) at $t=0,3,6$ for $A=0.6$ and $\lambda=1.5+\mathrm{i}$.

where

$$
\begin{aligned}
& E_{j, i}=-\left(1-2 D_{j, i}+D_{j, i} E_{j, i-1}\right)^{-1} D_{j, i}, \\
& F_{j, i}=\left(1-2 D_{j, i}+D_{j, i} E_{j, i-1}\right)^{-1}\left(H_{j, i}-D_{j, i} F_{j, i-1}\right),
\end{aligned}
$$

for $i=2,3, \ldots, N$ and

$$
E_{j, 1}=-\left(1-2 D_{j, 1}\right)^{-1} D_{j, 1}, \quad F_{j, 1}=\left(1-2 D_{j, i}\right)^{-1}\left(H_{j, 1}-D_{j, 1} H_{j, 0}\right) .
$$

Here we take the boundary condition

$$
H_{j, 0}=\left(\begin{array}{c}
0 \\
0 \\
Z_{j, 1}-\Delta s
\end{array}\right) .
$$

The values of all coefficients (6) are recursively determined by using (6) and (7) with (8). Taking $X_{j, N+1}=0, Y_{j, N+1}=0$ and $Z_{j, N+1}=Z_{j, N}+\Delta s$, we can solve the recurrence formula (5).

We have taken values of $\Delta s=1 / 8$ and $\Delta t=\Delta s^{3} / 32$. Number of steps $j$ is $12 / \Delta t$. Numerical accuracy is checked against the following aspects, i.e. i) the steady state vortex solution of (9) with $A=1$ propagates without showing significant distortion and ii) the conserved quantity [5] $\int\left(l_{s}-1\right) \mathrm{d} s$ conserves within the accuracy $5 \%$ for (1) and $10 \%$ for $(2)$.

\section{Initial value problem}

\subsection{Stretched vortex filament}

As an initial profile of the stretched vortex filament we take modified form of the one vortex soliton solution of (1) as

$$
\begin{aligned}
& X=A \frac{\lambda_{I}}{\lambda_{R}^{2}+\lambda_{I}^{2}} \sin (2 \Omega+\delta) \operatorname{sech}(2 \Theta+\epsilon), \quad Y=-A \frac{\lambda_{I}}{\lambda_{R}^{2}+\lambda_{I}^{2}} \cos (2 \Omega+\delta) \operatorname{sech}(2 \Theta+\epsilon), \\
& Z=s-\frac{\lambda_{I}}{\lambda_{R}^{2}+\lambda_{I}^{2}} \tanh (2 \Theta+\epsilon)
\end{aligned}
$$



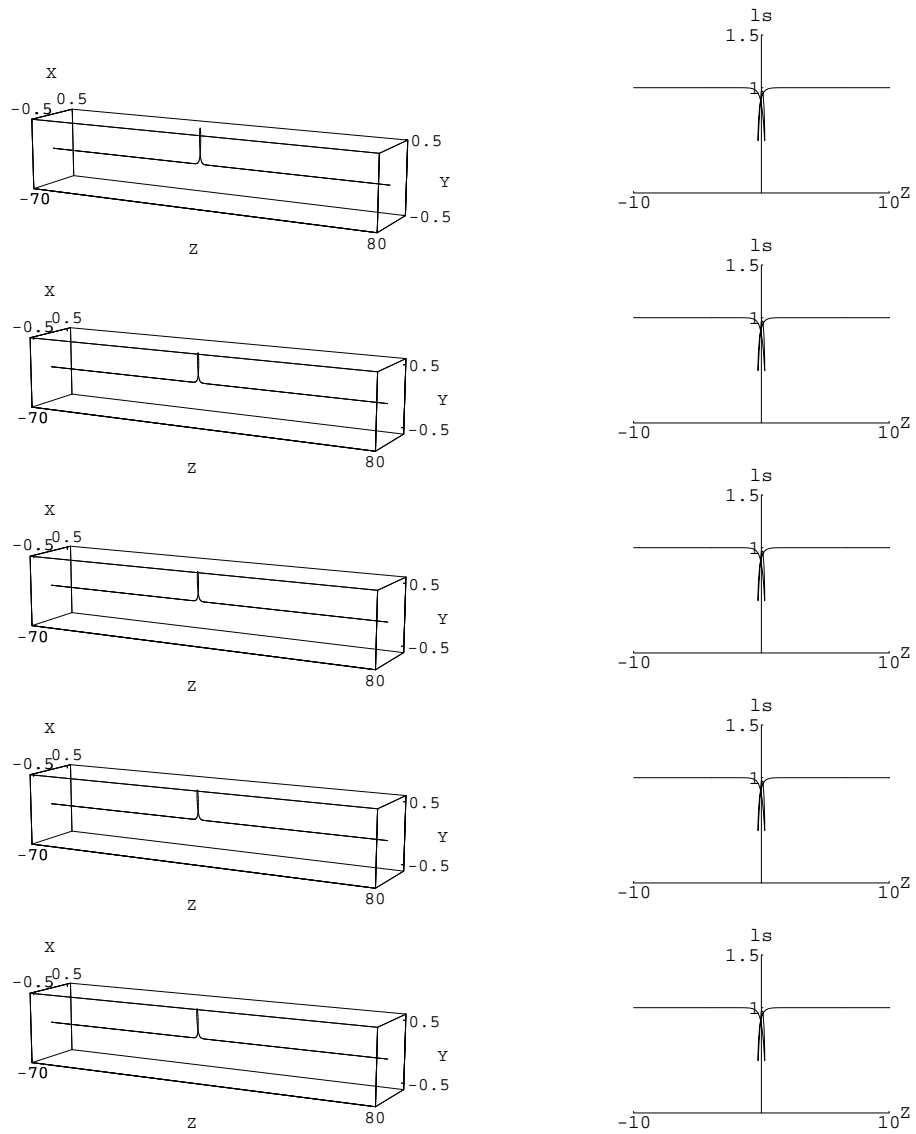

Figure 3. Time evolution (left) and local stretch (right) for the loop type of vortex soliton (10) of (1) at $t=0,3,6,9,12$ for $A=0.5$.

where $A$ is the factor to represent the stretch and we take $t=0$. If $A=1$, (9) is just the exact solution without the stretch. $\Omega, \Theta$ and constants $\delta, \epsilon$ are given by

$$
\Omega=\lambda_{R} s-\omega_{R} t, \quad \Theta=\lambda_{I} s-\omega_{I} t, \quad \tan \delta=-\frac{2 \lambda_{R} \lambda_{I}}{\lambda_{R}^{2}-\lambda_{I}^{2}}, \quad \epsilon=-\frac{1}{2} \log \frac{\left|c_{0}\right|^{2}}{4 \lambda_{I}^{2}},
$$

where $c_{0}$ is a constant. The wave number $\lambda=\lambda_{R}+\mathrm{i} \lambda_{I}$ and the frequency $\omega=\omega_{R}+\mathrm{i} \omega_{I}$ are connected by the dispersion relation $\omega=2 \lambda^{2}$. The local stretch is given by

$$
l_{s}^{2}=1+\frac{4 \lambda_{I}^{2}}{\lambda_{R}^{2}+\lambda_{I}^{2}}\left(A^{2}-1\right)\left[\operatorname{sech}^{2}(2 \Theta+\epsilon)-\frac{\lambda_{I}^{2}}{\lambda_{R}^{2}+\lambda_{I}^{2}} \operatorname{sech}^{4}(2 \Theta+\epsilon)\right] .
$$

If $A=1$, there is no stretch. If $A \neq 1,(9)$ shows the local stretch for $A>1$ and the local shrink for $A<1$.

Using LIE, we show the profiles and the local stretch of the vortex filament in Fig. 1 for $A=0.6$ with $\lambda=1.5+\mathrm{i}$ and $c_{0}=1$. We observe that, since the filament has shrunk initially, then the vortex soliton deforms its profile and moves away with the strong radiation, however, the shrunk region remains in the same place as freezing the shrink [1].

For the case of the equation of motion (2), we illustrate the profiles and the local stretch of the filament in Fig. 2 with the same parameters such as $A=0.6, \lambda=1.5+\mathrm{i}$ and $c_{0}=1$. Comparing with LIE, we observe that the vortex soliton takes a small modification and moves fast with the weak radiation. The shrunk region remains in the same place, which is similar to the case of LIE. 

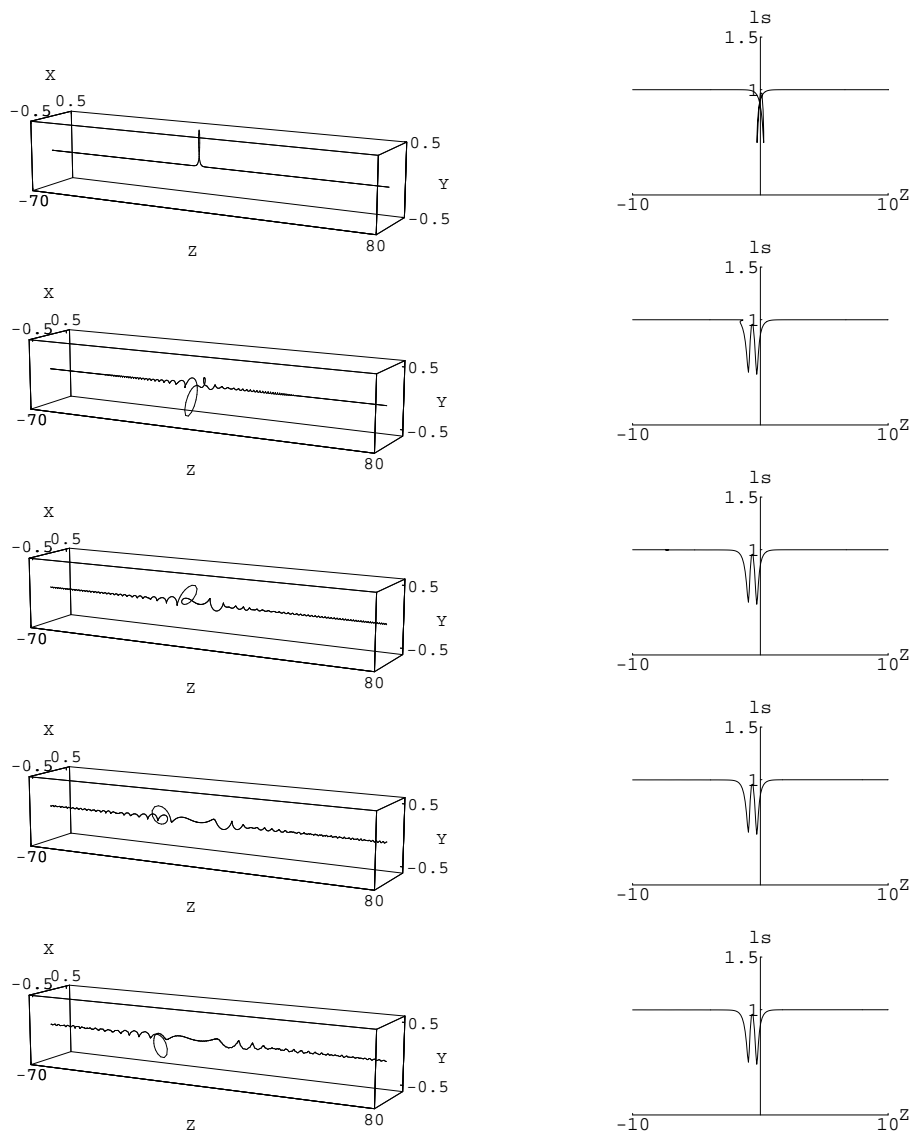

Figure 4. Time evolution (left) and local stretch (right) for the loop type of vortex soliton (10) of (2) at $t=0,3,6,9,12$ for $A=0.5$.

\subsection{Stretched loop type vortex filament}

With the exact solution of (1) as

$$
X=-A \sin 4 t \operatorname{sech} 2 s, \quad Y=A \cos 4 t \operatorname{sech} 2 s, \quad Z=s-\tanh 2 s,
$$

we consider the initial value problem by taking $t=0$. Here $A$ is the factor to represent the stretch for the loop type of the vortex filament. The local stretch is given by

$$
l_{s}^{2}=1+4\left(A^{2}-1\right) \operatorname{sech}^{2} 2 s \tanh ^{2} 2 s .
$$

In Fig. 3 we show the profiles and the local stretch of the loop type of the vortex filament for $A=0.5$ at the instants $t=0,3,6,9,12$ by using (1). Since the initial profile is the exact solution we observe that the vortex filament rotates in the same place with a constant angular velocity and that the shrunk region does not move [1].

Using the evolution equation (2), we simulate the vortex filament with the same parameter as $A=0.5$ and show the profiles and the local stretch at the instants $t=0,3,6,9,12$ in Fig. 4. From the profiles, we can observe that new propagating vortex soliton is produced by accompanied with the radiation. However, from the local stretch, we find that shrunk region with double dips remains in the nearly same place, which means that the mode with a constant $X$, a constant $Y$ and two kinks for $Z-s$ excited in the region. 


\section{Conclusion}

We have presented the numerical scheme and considered the initial value problem in order to stress the difference of two equations (1) and (2). We have found that the profiles of the vortex filaments show quite different behaviour, but for the local stretch, both of the two equations give the same results in such a way that the initial stretched region stays in the same place to freeze the stretch or the shrink.

[1] Konno K., Kakuhata H., A new type of stretched solutions excited by initially stretched vortex filaments for the local induction equation, Theor. Math. Phys., 2005, V.144, 1181-1189.

[2] Arms R.J., Hama F.R., Localized-induction concept on a curved vortex and motion of an elliptic vortex ring, Phys. Fluids, 1965, V.8, 553-559.

[3] Konno K., Kakuhata H., Stretching of vortex filament with corrections, in Nonlinear Physics: Theory and Experiment, II (2002, Gallipoli), River Edge, NJ, World Scientific Publishing, 2003, 273-279.

[4] Hashimoto H., A soliton on a vortex filament, J. Fluid Mech. 1972, V.51, 477-485.

[5] Konno K., Kakuhata H., A hierarchy for integrable equations of stretched vortex filament, J. Phys. Soc. Japan, 2005, V.74, 1427-1430.

[6] Konno K., Mitsuhashi T., Ichikawa Y.H., Dynamical processes of the dressed ion acoustic solitons, J. Phys. Soc. Japan, 1977, V.43, 669-674. 